\title{
Towards Automated CALibration of Visible Light POSITIONING SYSTEMS
}

\author{
Robin Amsters \\ KU Leuven, \\ Department of Mechanical Engineering, \\ 3000 Leuven, Belgium \\ robin.amsters@kuleuven. be \\ Nobby Stevens \\ KU Leuven, \\ Department of Electrical Engineering, \\ 3000 Leuven, Belgium \\ nobby. stevens@kuleuven. be
}

\author{
Eric Demeester \\ KU Leuven, \\ Department of Mechanical Engineering, \\ 3000 Leuven, Belgium \\ eric.demeester@kuleuven. be \\ Peter Slaets \\ KU Leuven, \\ Department of Mechanical Engineering, \\ 3000 Leuven, Belgium \\ peter.slaets@kuleuven. be
}

\begin{abstract}
Localization based on visible light is a novel technique for indoor positioning that provides a number of advantages over traditional radio frequency based approaches. An important step in the deployment of visible light positioning systems is the calibration procedure, during which environmental parameters such as the positions of light sources are determined. This work presents a proof-of-concept approach to obtain these parameters in an efficient manner by using a mobile robot. This robot builds a map of the environment, and adds the location and identifier of optical transmitters to this map. With this approach, light source modulation frequencies can be estimated with sufficient accuracy to uniquely identify each source. Additionally, the inter-LED distance has an average accuracy of less than $10 \mathrm{~cm}$ compared to the real distance.
\end{abstract}

Keywords Visible Light Positioning · Indoor Positioning · Calibration · Simultaneous Localization and Mapping

\section{Introduction}

Accurate and cost-effective indoor positioning is still an open research challenge. Contrary to outdoor spaces, indoor environments can have vastly different layouts. For example, a warehouse typically consists of large open spaces, whereas a typical office building contains much more walls. This variety in indoor layouts has lead to an equally wide variety of indoor positioning systems (IPS), each with their unique advantages and disadvantages. In recent years, positioning based on visible light has become more interesting due to the increased adoption of solid state lighting. The LED lighting market is even expected to surpass 100 billion USD in 2024 [1]. In visible light positioning (VLP), the visible light spectrum is used as a transmission medium. Light intensity is modulated at frequencies that are imperceptible to the human eye, yet can still be picked up by a photodiode or camera, thus enabling wireless communication. Generally, light fixtures in a VLP system emit some sort of identifier, such as a unique code or frequency [2]. The coordinates corresponding to each light source can be emitted as part of the signal, or stored at the receiver side with a link to the identifier [3] (for example, a modulation frequency of $2 \mathrm{kHz}$ could correspond to a light source at the beginning of a particular hallway). VLP systems can often reuse existing lighting infrastructure, thus lowering the investment and invasiveness of such systems. Additionally, accuracy can be as low as a few centimeters [4]. 
While VLP systems have many advantages, information on the light fixture deployment is a prerequisite. Sometimes, building maps can provide the positions of fixtures in a building. However, these maps are not necessarily accurate. Additionally, these maps do not contain the light source identifiers. Therefore, a calibration procedure is still required. Such a procedure would be less labor-intensive than, for example, RF fingerprinting, as a dense signal grid is not required. However, for large deployments, manual calibration is not desirable, especially when building maps are not available. In this work, we describe how a mobile robot can be used as a calibration device for VLP systems. The robot performs simultaneous localization and mapping (SLAM) to build a map of the environment based on data from a 2D laser scanner. Additionally, a ceiling-facing camera detects nearby light sources and their frequencies. The combination results in a map of the environment, with the light source locations and their unique identifiers. This information is then ready to be used by VLP systems.

The rest of this work is structured as follows. Section 2 discusses related work. In Section 3, a system description is provided. Experimental results are presented in Section 4 and discussed in Section 5. Finally, a conclusion is drawn in Section 6.

\section{Related work}

SLAM algorithms generally aim to reconstruct a map of the environment while simultaneously estimating the trajectory of the observer relative to that map. A number of solutions have been proposed over the years, the most common approaches are based on Bayesian filtering [5]. A number of algorithms are also freely available in frameworks such as the Robot Operating System (ROS) [6]. One such algorithm (Google Cartographer [7]) was used in this work (see Section 3).

One application where robots can significantly reduce deployment time and cost, is in building a signal database for fingerprinting-based IPS. These systems generally operate in two stages. In the first (off-line) stage, location-dependent features (known as fingerprints) are collected at known locations and are stored in a database. These features are usually the received signal strength of some RF signal, but camera images can also be used as features. During the second (on-line) stage, measurements are matched to the database to obtain a position estimate. Methods such as kNN, neural networks and support vector machines can be used to match the received signal to the database [8]. In previous works, robot platforms have already been employed to collect the signal fingerprints. For example, in WIFI [9][10] and RFID-based positioning systems [11][12].

A large body of literature is available on the subject of channel modeling for VLP [13][14][15][16]. Such models are often used to relate received light intensity to a transmitter-receiver distance. When the transmitter locations are known, these distances can be used to determine the position of the receiver via triangulation [4]. Most publications do not describe how the light source locations should be obtained. Signify (previously Philips lighting) recently concluded a project where a mobile robot was used to map a stadium, in order to determine where light fixtures should be placed [17]. However, the problem of obtaining light fixture identification data of an existing deployment has, to the best of our knowledge, not been addressed.

\section{System description}

We perform the calibration procedure for a small-scale VLP setup with 4 LEDs modulated at different frequencies (Fig. 1). We use this setup to illustrate the proof-of-concept, yet the approach is scalable to larger environments. On-off keying is used as a modulation technique, that is, lights are either completely on or off. The modulation frequencies are used as the LEDs unique identifier, and range from approximately $1.5 \mathrm{kHz}$ to $5 \mathrm{kHz}$. Frequency selection is elaborated in Section 3.2. A mobile robot is used as a calibration platform. On top of the robot platform, a 2D laser scanner is mounted (see Fig. 2). Scanner data are used by the Google Cartographer SLAM algorithm [7]. Additionally, the robot is equipped with a ceiling-faced camera. We use the OpenMV H7 [18] because of the high degree of control it allows compared to a normal webcam. During experiments, the robot is manually driven to each light source, and records data for a few seconds while the LED is approximately in the center of the image.

Conventional cameras generally have a frame rate of 30 or 60 frames per second, which may seem insufficient to sample VLP signals that can be in the $\mathrm{kHz}$ range. However, most CMOS cameras read out the lines of the sensor sequentially, this is known as a 'rolling shutter'. This technique can be used to produce relatively low-cost cameras, yet can produce some undesirable effects such as a skewed image if the camera is moving. However, in VLP systems, it can be exploited to sample high-frequency light modulation. The use of this principle has been explored for standalone CMOS cameras 


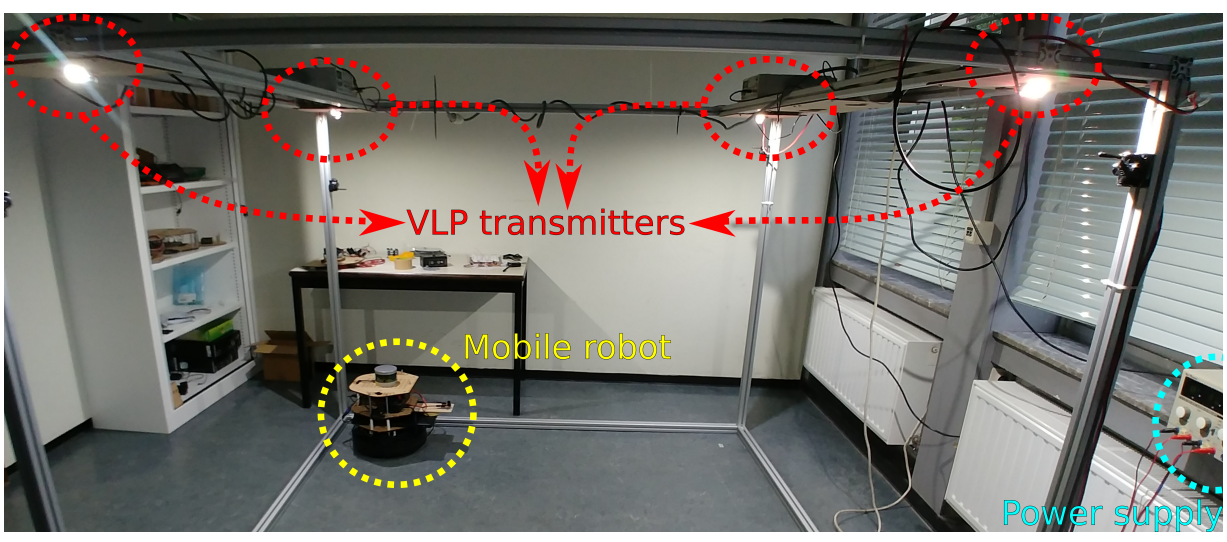

Figure 1: Experimental setup

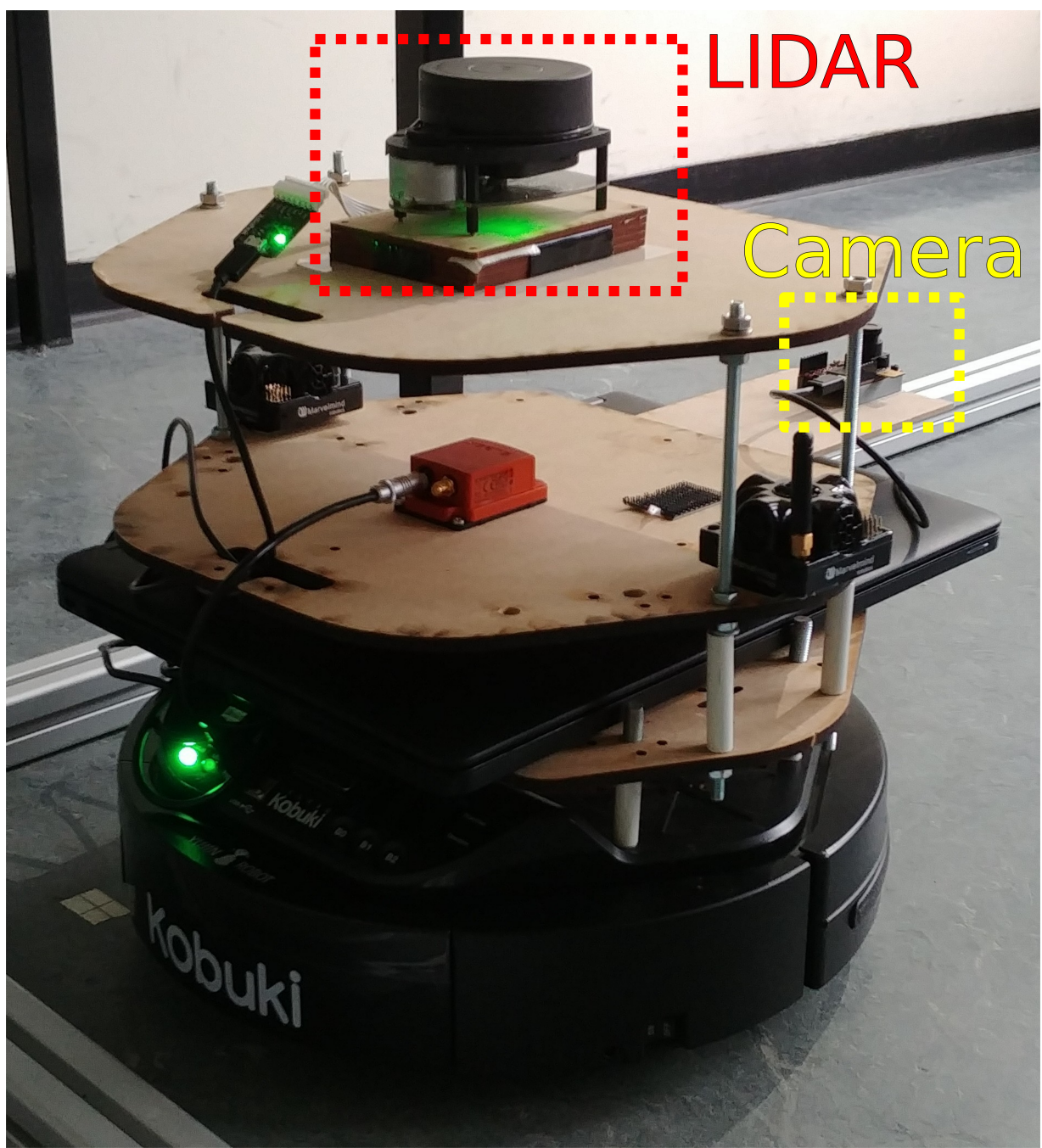

Figure 2: Calibration platform with 2D laser scanner. The other sensors on the lower platform are not used in this work

[19] and smartphones [3] for localization and communication purposes. A schematic representation of the rolling shutter principle is provided in Fig. 3a. The top row in the illustration represents the status of the LED over time, which is either at nominal intensity or completely off. The currently exposed rows of the image sensor will represent the 
captured intensity of the incident light. Sequentially exposed rows capture the changes in light intensity, enabling the decoding of information. Fig. $3 \mathrm{~b}$ shows an example image of a modulated light source captured with a rolling shutter.

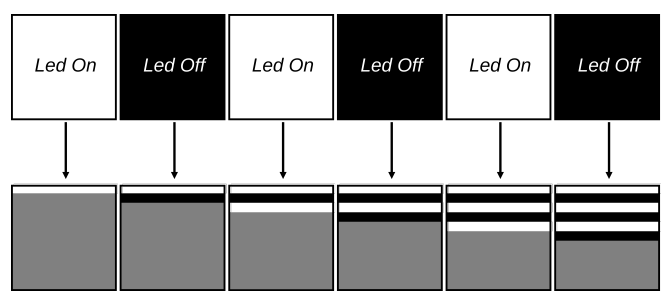

(a) Rolling shutter principle

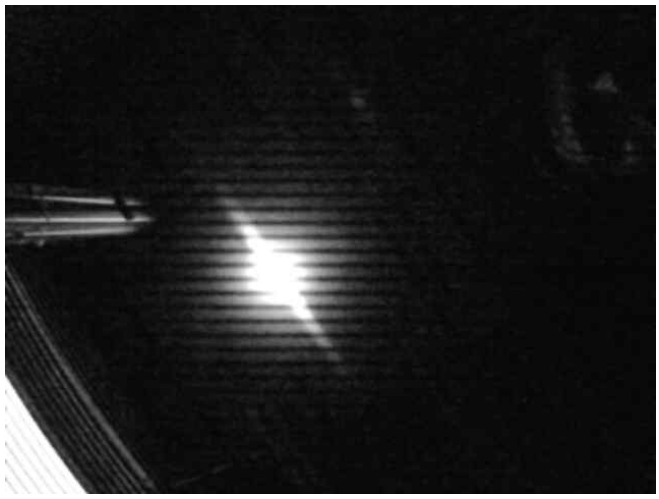

(b) Picture of a modulated light source captured with a rolling shutter

Figure 3: Rolling shutter for visible light positioning

\subsection{Algorithm overview}

All data processing is performed offline, as a calibration procedure generally only needs to be performed a limited number of times. For example at the time of installation, or following renovations. Therefore, there is little gain from performing the calibration online. Conceptually, the algorithm works as follows. From Cartographer, we obtain a map of the environment, as well as the robot trajectory. The image processing algorithm returns coordinates of the detected light source in the camera frame, as well as the modulation frequency (see Section 3.3). By combining the map of the environment, the robot trajectory and the light coordinates with associated frequency, we obtain a map of the environment that contains the 2D light source coordinates with their identifiers (see Section 3.4). The height of the ceiling and the total number of lights are assumed to be known. The light source coordinates and their modulation frequencies can later on be used by VLP systems. The map could be used in an application, for example to show the user's location. Additionally, system accuracy could be improved by employing map matching. In this approach, location hypotheses that do not correspond to the map (e.g. cross walls) can be removed [20]. Fig. 4 illustrates the flow of data. The Cartographer source code is freely available online ${ }^{1}$, a detailed description of the algorithm can be found in [7]. The image processing workflow, and the combination with the output of SLAM are discussed in Sections 3.3 and 3.4 , respectively.

\subsection{Frequency selection}

Every transmitter has a unique frequency, that serves as its identifier during positioning. Thus, frequencies need to be distinct, yet have enough separation to avoid ambiguities. One approach would be to select frequencies at evenly spaced intervals. However, the stripe height (see Fig. 3b) in the image is proportional to the signal period. As the signal period is the inverse of the frequency, an even spacing of frequencies does not result in an even spacing of stripe heights. Better results can be obtained when the transmitter frequencies are selected based on the difference in period length. Additionally, transmitters and receiver are not time synchronized in this setup. The schematic overview of the rolling shutter effect in Fig. 3a aligns the transition edges of the emitted signal with the start of row exposures in the image sensor. This is not necessarily the case, partially exposed rows (where the light is toggled on or off during the exposure period) show less than the maximum intensity. Partial exposure can in turn lead to over- or underestimation of the stripe length in image processing. Estimation errors can be minimized by choosing signal periods as integer multiples of the line readout time $t_{\text {line }}$ of the camera. In that case, opposite signal transitions occur at equal shifts relative to the start of row readout. Ideally, the period of the LED transmitter signal is aligned with the row readout of the image sensor. Rows are exposed under constant light intensity, and no row experiences a signal transition during its exposure time. This situation is shown at the top of Fig. 5. In the examples below, a positive and negative time shift $d t$ between periods is introduced. Signal periods are integer multiples of the readout time $t_{\text {line }}$, therefore the shift only affects two rows per period. These are partially exposed, and one row receives less light while another captures more light

\footnotetext{
${ }^{1}$ https://github.com/googlecartographer
} 


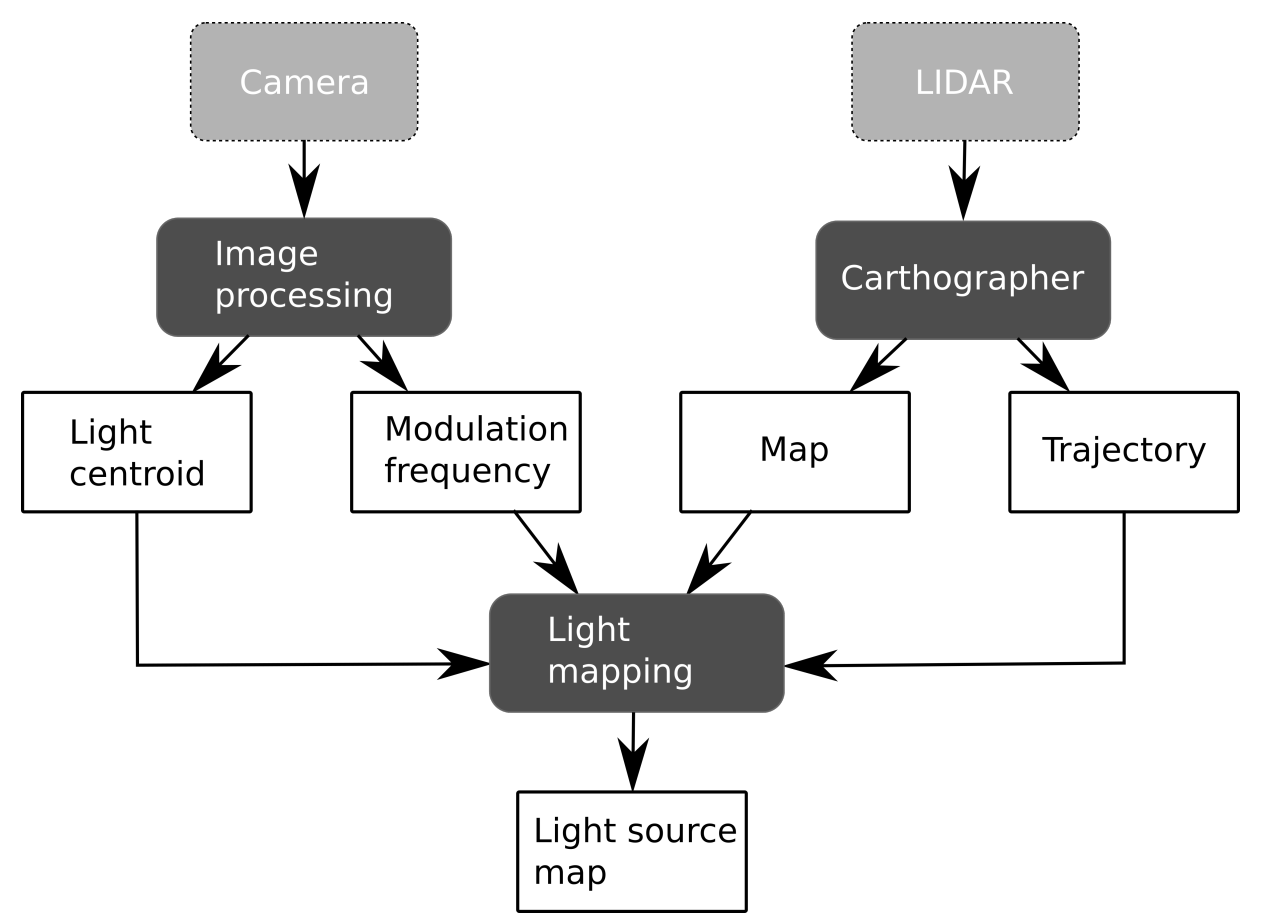

Figure 4: Schematic representation of the proposed algorithm

Table 1: Selected transmitter frequencies

\begin{tabular}{|l|l|l|}
\hline $\begin{array}{l}\text { Pixels per } \\
\text { Period [px] }\end{array}$ & Period $[\mu$ s] & Frequency [Hz] \\
\hline 22 & 638,8 & 1565,4 \\
17 & 493,6 & 2025,8 \\
12 & 348,4 & 2869,9 \\
7 & 203,3 & 4919,8 \\
\hline
\end{tabular}

than the ideal case. If the classification threshold for bright and dark rows is set to the mean value of minimum and maximum intensities, rows are only classified as bright if exposed for at least half a readout period. As a result, the number of rows classified as bright or dark do not change. Even in the limiting case of $d t=\frac{t_{\text {line }}}{2}$ (shown at the bottom of Fig. 5), the period can be correctly determined, as both affected rows would be classified as bright. This would result in the bright stripe being considered one row thicker, whereas the dark stripe's height is underestimated by one row. Consequently, the average of both still results in the same value, from which the period and frequency of the transmitter signal can be identified. This property also explains why it is sufficient that the signal period is an integer multiple of $t_{\text {line }}$, even if the half period length is not.

In order to select the final transmitter frequencies, the usable frequency range was first determined. For this purpose, images of a single transmitter were captured with increasing modulation frequencies. Frequencies in the range of 1.5 $\mathrm{kHz}$ to $5 \mathrm{kHz}$ could be identified by the algorithm. At lower frequencies, the image contains insufficient stripes for identification, while at higher frequencies the stripes are of insufficient height to be separable. Within this range, four frequencies were selected, such that the periods are integer multiples of $t_{\text {line }}$, yet that the whole operating range is represented. These frequencies are shown in Table 1. The conversion from stripe heigth to transmitter frequency is performed as follows:

$$
f_{t x}=\frac{1}{p_{t x}}=\frac{1}{n_{p x, \text { stripe }} t_{\text {line }}}
$$

where $f_{t x}$ is the transmitter frequency, $p_{t x}$ is the period of the signal, $n_{p x, \text { stripe }}$ is the number of pixels per stripe and is the line $t_{\text {line }}$ readout time (approximately $29 \mu$ s for our camera) 


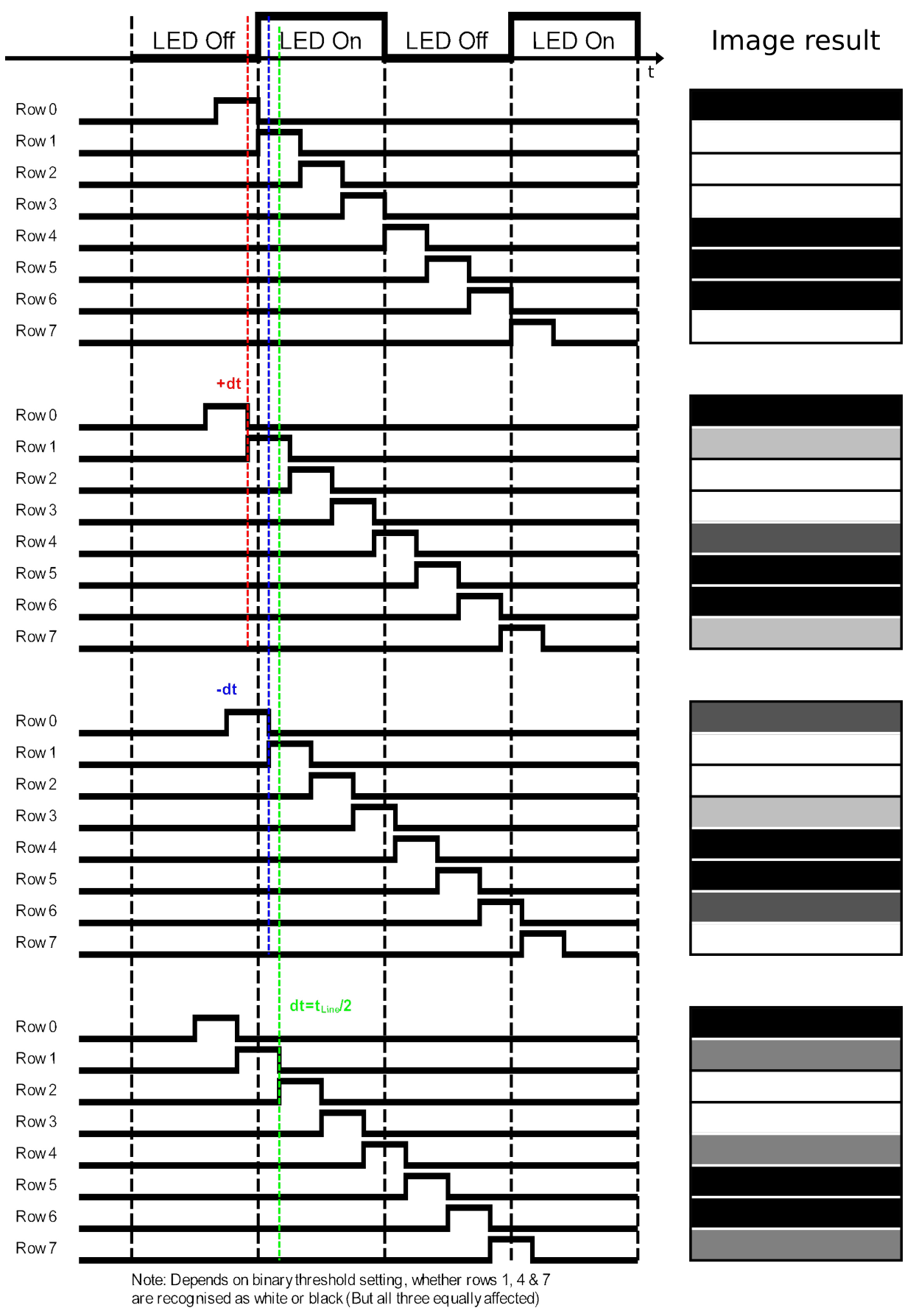

Figure 5: (top) Signal capture with ideal alignment of signal and readout periods. (second from top) Signal capture with a positive time shift of $d t$. (third from top) Signal capture with a negative time shift of $d t$. (bottom) Signal capture a time shift of $\frac{t_{\text {line }}}{2}$ between signal and readout

\subsection{Image processing}

The image processing pipeline is illustrated in Fig. 6 for an LED with a modulation frequency of approximately 2 $\mathrm{kHz}$. This pipeline is based on the work by Kuo, Pannuto, Hsiao and Dutta in [3]. The original image is first blurred, to remove the stripe pattern and reveal the light source location. A binary threshold is then applied, and the center of the blob is located. The coordinates of this point are assumed to coincide with the center of the light source. These 
coordinates are one input for the light mapping algorithm (see Section 3.4). Additionally, they are also used to crop the original image around the center-point of the light source. This cropping operation is intended to increase efficiency, as it results in a smaller image to be processed in the next steps. A Gaussian blur is also applied to the cropped image to make the stripe pattern more visible. Next, the contours of the stripes are retrieved via Canny edge detection [21], and the number of pixels between a transition from a bright to a dark line are counted. One bright line and one dark line correspond to one signal period. Therefore, the line readout time and the number of vertical pixels in the bright and dark lines can be used to calculate the modulation frequency.

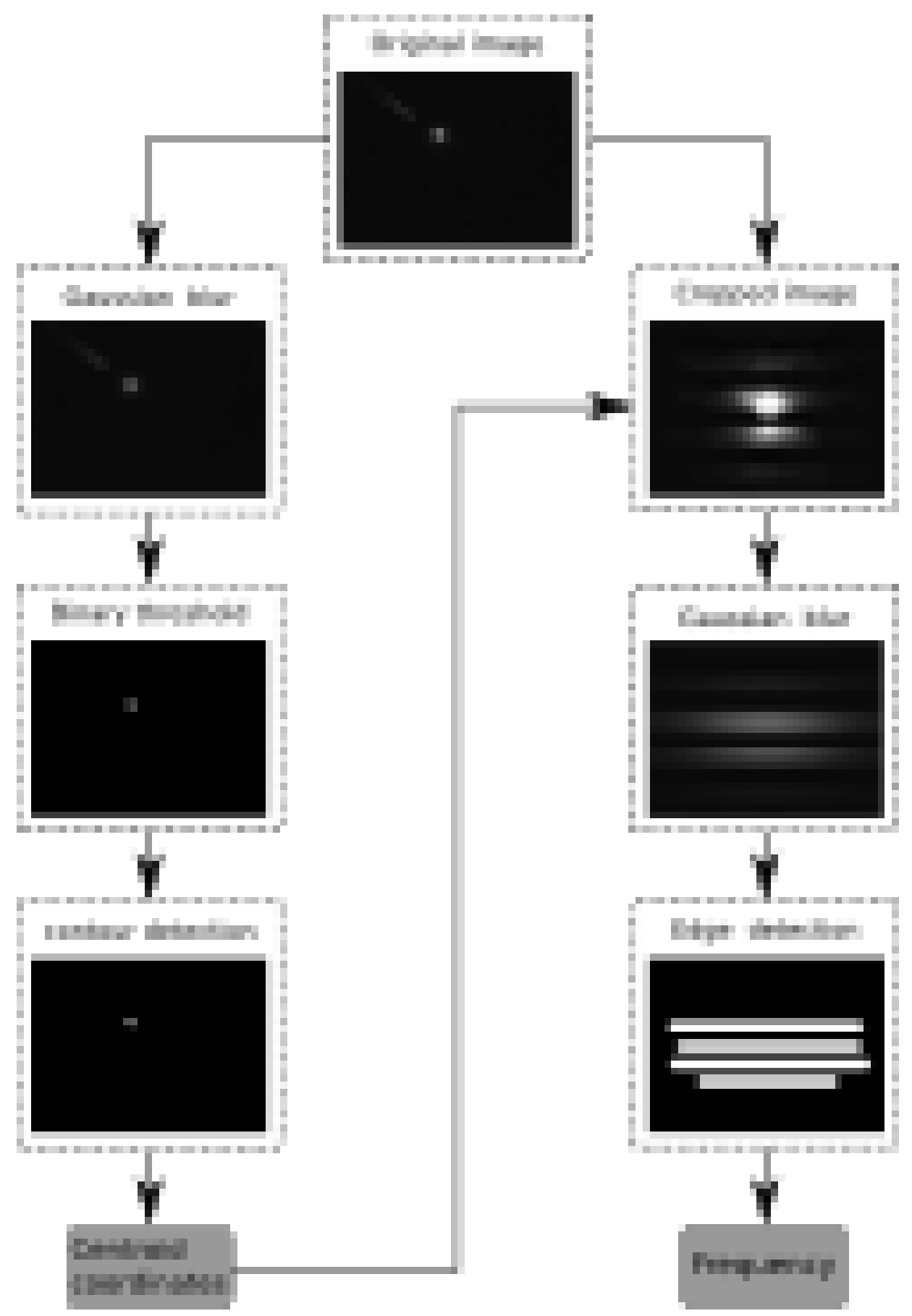

Figure 6: Image processing pipeline 


\subsection{Light mapping}

After an experiment has concluded, we loop over all the captured images, and extract the light source frequencies. We use only the images where a light source was detected close to the center, as distortion is usually lowest in this region. The result is a spectrum such as Fig. 7. In this figure, the $y$-axis represents the number of pictures where a certain frequency was detected. One would expect to only see 4 peaks in this spectrum, corresponding to the modulation frequency of the LEDs. However, the camera motion introduces some motion blur, and therefore a calculation error. The direction of motion may also have an impact on the frequency detection. For example, a light source that moves vertically across the image may experience different degrees of motion blur than a light source that moves diagonally across the image. As the final identified frequency, we take the 4 highest peaks in the spectrum. Next we loop over the images again, and extract only the coordinates of the centroids that correspond to one of the identifiers. Again we only use the coordinates that are detected close to the image center to minimize distortion. At this stage, we have one time-stamped dataset, namely the light coordinates in the image with the corresponding frequency. Next, the SLAM algorithm is used to produce a map and a time-stamped trajectory. The trajectory is synchronized with the image processing data, and only the samples from which the time-stamps in both arrays approximately match, are considered in the next step. By using the robot trajectory and the relative position of the camera to the robot center, it is possible to translate the motion of each unique light source from the image frame to the map frame. Ideally, this results in a single point on the map for each light source. Instead, a small spread was observed on the light source coordinates. The average of all detected locations was used as the final result.

\section{Spectrum}

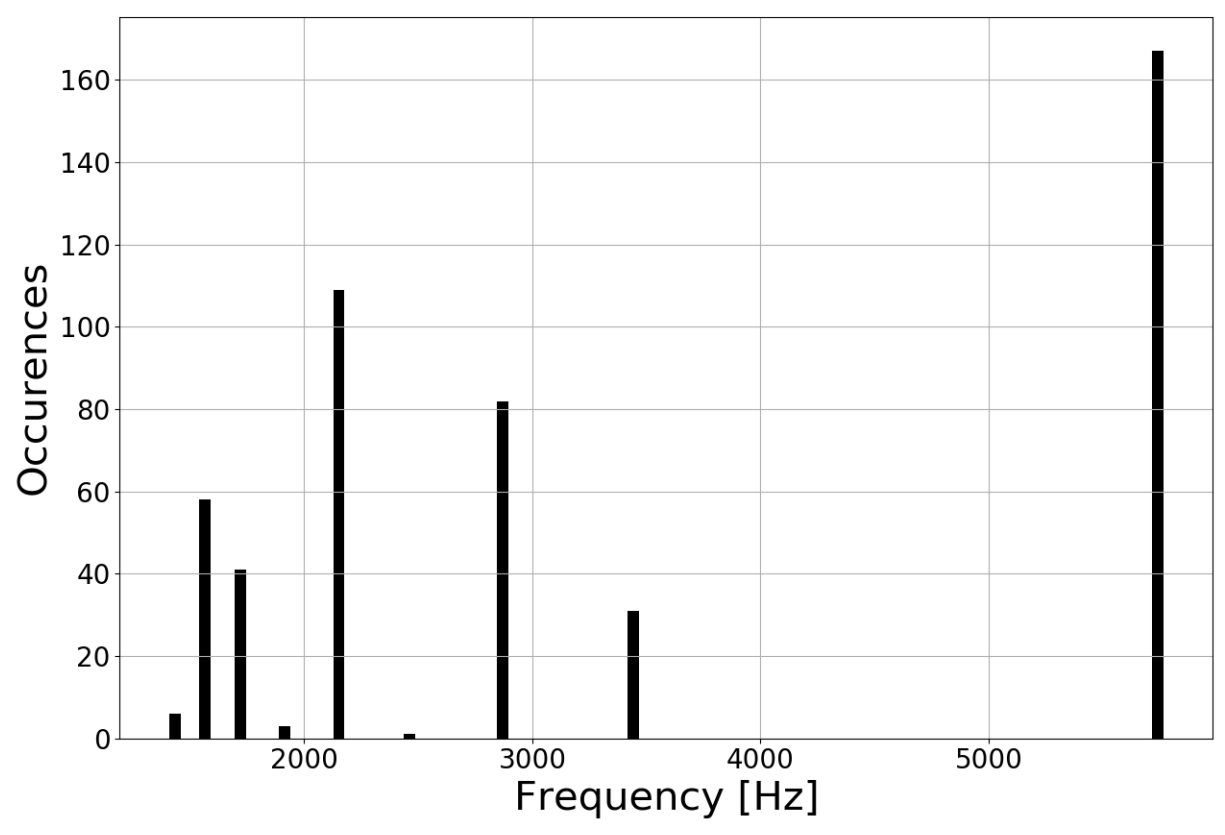

Figure 7: Example spectrum recorded during an experiment

\section{Experimental results}

\subsection{Frequency detection results}

To evaluate the accuracy of the frequency detection step, experiments were first performed with a static receiver. The camera was placed underneath every transmitter, and 6 pictures were taken while the camera remained static directly below the LED. The observed estimation accuracy was approximately the same for all tested frequencies. However, the higher frequency signals tended to produce slightly better results. This can likely be attributed to the fact that a higher modulation frequency results in more stripes in the image. Therefore, the average frequency estimate can be more accurate. Fig. 8 shows the cumulative distribution of frequency estimation errors. On average, modulation frequencies can be recovered with an accuracy of less than $40 \mathrm{~Hz}$. The vast majority of frequencies (87\%) can be recovered with an 
Table 2: Frequency detection results

\begin{tabular}{|l|l|l|}
\hline $\begin{array}{l}\text { Applied } \\
\text { frequency }[\mathrm{kHz}]\end{array}$ & $\begin{array}{l}\text { Detected } \\
\text { frequency }[\mathrm{kHz}]\end{array}$ & Error $[\mathbf{k H z}]$ \\
\hline 1.57 & 1.57 & 0.00 \\
2.03 & 2.15 & 0.13 \\
2.87 & 2.87 & 0.00 \\
4.92 & 5.74 & 0.82 \\
\hline
\end{tabular}

accuracy of $70 \mathrm{~Hz}$ or less.

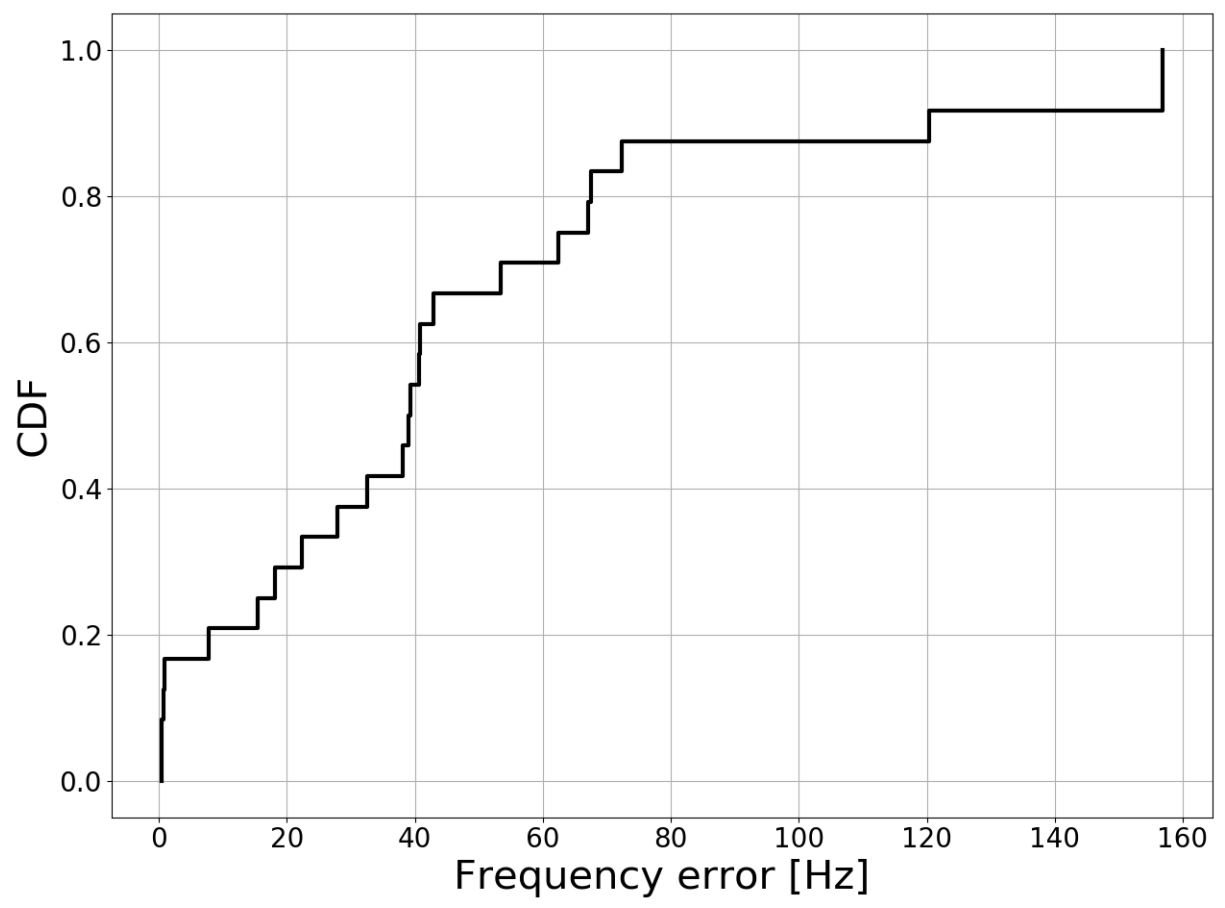

Figure 8: Cumulative distribution of frequency errors for static receiver

Experiments have also been performed while the camera was mounted on the moving robot platform. A fair evaluation requires that an equal amount of pictures are taken of every transmitter while the robot is moving. Additionally, the movement speed should be equal when passing every light source. Such conditions are challenging to create, therefore only the end result of the calibration procedure is evaluated here.

Table 2 compares the frequencies estimated by the calibration procedure to the real modulation frequencies. It is clear that the lower frequencies can be obtained relatively easily. The LEDs that were modulated at $1.57 \mathrm{kHz}$ and $2.87 \mathrm{kHz}$ could be identified almost exactly (deviation significantly lower than $1 \mathrm{~Hz}$ ). The frequency of one LED had a deviation of approximately $125 \mathrm{~Hz}$. However, the LED modulated at approximately $5 \mathrm{kHz}$ could not be identified accurately. The frequency estimation error was more than $800 \mathrm{~Hz}$ in this case. The experiment was repeated two more times, and the same results were obtained each time.

\subsection{Light mapping results}

Fig. 9 shows an example map and robot trajectory returned by the SLAM algorithm. The probability that a certain cell is part of an obstacle, is indicated by its intensity. Gray areas are unexplored, and become brighter or darker depending on the observations made by the robot. A black pixel is identified as an obstacle, while white pixels correspond to free space. The room was not completely empty during testing, therefore some parts of the room were not visible to the laser scanner and remain dark gray. The light sources with their identified frequencies are also present on this map. The LEDs were mounted in a construction consisting of aluminum ITEM ${ }^{\circledR}$ profiles (see Fig. 1.). The vertical poles were 
detected by the laser scanner and are highlighted on the map. The LEDs are mounted inside the square defined by these poles. At first glance, the estimated locations appear to be approximately correct. However, it is challenging to compare

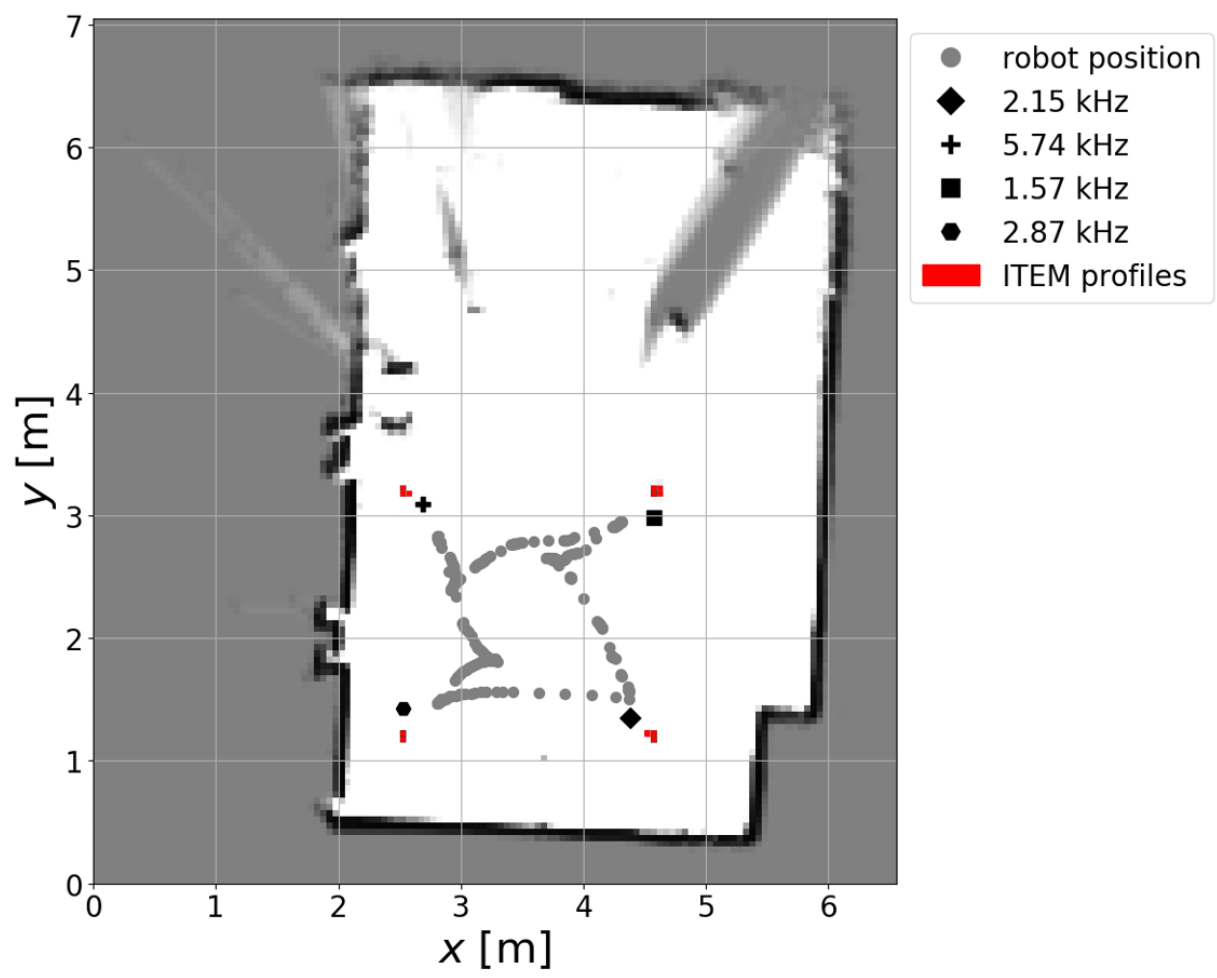

Figure 9: Map with detected light sources.

the obtained LED coordinates with their true locations in the test setup, as the transformation between the coordinate frames is not known. As a quantitative measure of accuracy, we can use the distances between the LEDs, as these are independent of the coordinate frames. Therefore we calculate the distance between every LED, which is essentially the length of the sides and diagonals of the square defined by the LED coordinates. The difference between the distances obtained from the calibration procedure, and the distances measured manually in the experimental setup are shown in Fig. 10. This figure shows the combined results from all experiments as a cumulative distribution. On average, the estimation error is approximately $10 \mathrm{~cm}$. Out of all the distance measurements, $75 \%$ have an accuracy of less than 15 $\mathrm{cm}$. Larger intervals of the distribution show that in some cases, the estimation error can be as high as $25 \mathrm{~cm}$. This can likely be attributed to the low frequency LED, which in some experiments has a larger offset than the others. An example of such a map is shown in Fig. 11. The low number of stripes makes it more challenging to accurately locate the LED centroid. This inaccuracy is propagated to the final LED location in the map.

\section{Discussion}

The camera resolution and the image processing place restrictions on the frequencies that can be identified. On the other hand, it is possible to re-use frequencies in a typical VLP setup, as every transmitter has a relatively small coverage area and signals cannot cross walls. Experiments with a stationary receiver result in a high accuracy for the selected frequencies. The movement of the robot does most likely introduce extra ambiguities in the frequency estimation step, which become particularly prominent at high frequencies. At high frequencies, the lines in the image become relatively thin, so a detection error of a few pixels results in a large frequency error. The obtained results with a dynamic receiver are still sufficiently accurate to uniquely distinguish transmitters of most VLP systems. The error in the detected frequency is usually lower than $150 \mathrm{~Hz}$. VLP transmitters are generally spaced further apart in the frequency spectrum. At high frequencies, the estimation procedure does deteriorate. Nevertheless, $5 \mathrm{kHz}$ is already a relatively high frequency. Most VLP systems operate on significantly lower frequencies.

The accuracy of the generated map may be insufficient for highly demanding applications, such as robot positioning. Nevertheless, many applications can be envisioned for lower accuracy IPS. In early 2016, the Open Geospatial 


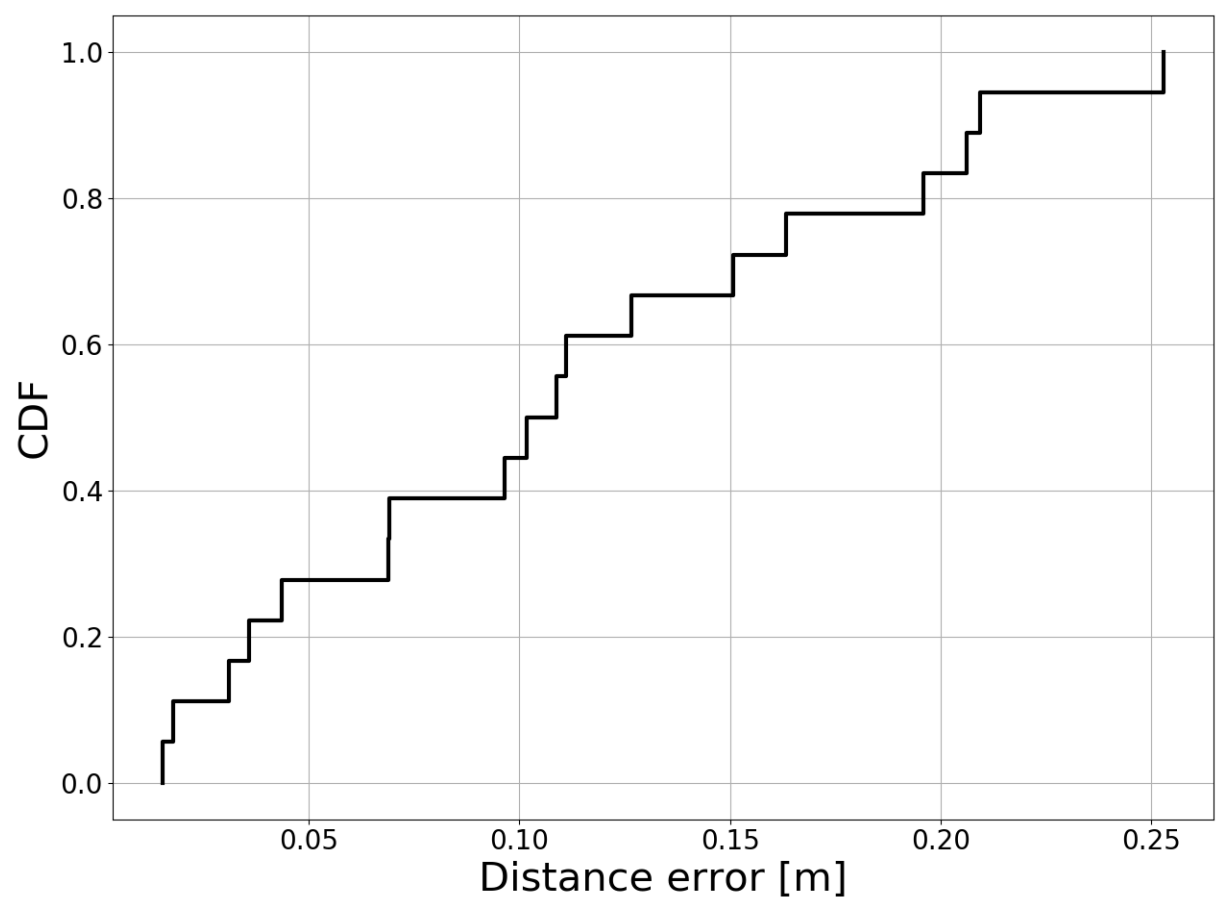

Figure 10: Cumulative distribution of distance estimation errors from all experiments

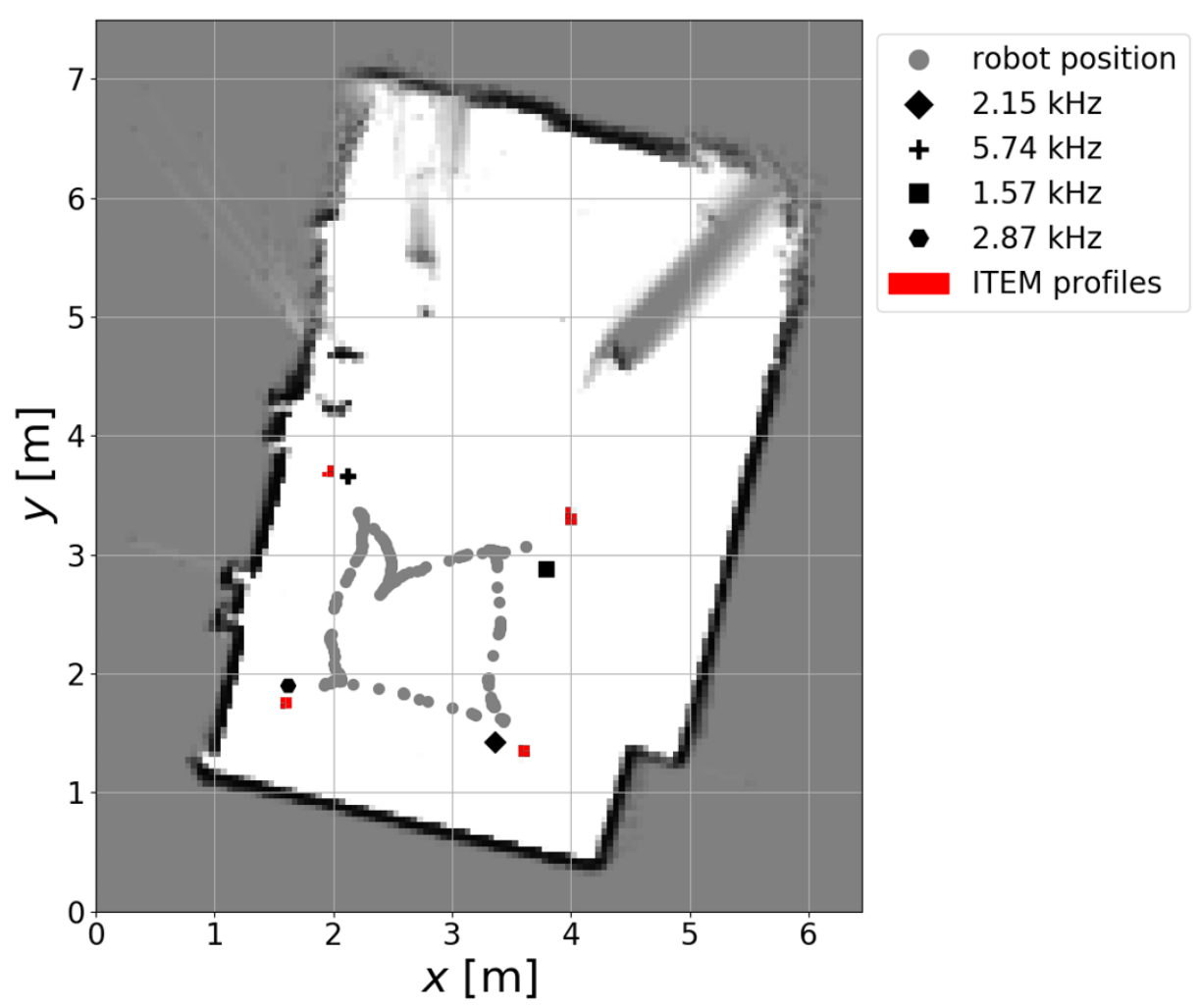

Figure 11: Light map with erroneous estimation of the high frequency LED position

Consortium (OGC), the InLocation Alliance and the European i-locate project jointly launched a survey on the benefits of indoor localization. Responses were received from 153 client organizations and technology suppliers in 33 countries. 
Respondents indicated that an accuracy of $1 \mathrm{~m}$ is sufficient for most of their needs [22]. Additionally, the survey concluded that "from a hardware standpoint instead focus should be paid to development of low-latency indoor location technologies capable of collecting data from passive or very low power devices, and to the development of solutions that are simple to deploy and configure, ideally allowing clients to install them autonomously (e.g., through self-configuration means)" [22]. VLP systems can already provide low-latency indoor location, and can be built with relatively low power devices. This work demonstrates that the deployment of future systems could be simplified as well.

\section{Conclusion}

This proof of concept work outlines a methodology to simplify the calibration procedure of VLP systems. By combining an open source SLAM algorithm with rolling shutter based frequency detection, the location of light sources and their identifiers can be obtained. Frequency detection is sufficiently accurate to uniquely distinguish all light sources. The generated light source map can be used by VLP systems that have modest accuracy requirements.

In future work, significant improvements could be made by using a higher resolution camera. Due to the higher sampling rate, the frequency detection would most likely increase in accuracy. Moreover, a higher resolution provides better granularity for the light mapping. In this paper, the robot was driven manually. However, one could also investigate the option of autonomous exploration by the robot platform, to further increase efficiency. Our calibration procedure also does not determine the intensity or height of detected light sources, which are required by some received signal strength positioning approaches. The proposed approach could be extended to obtain this information as well. For example, the light intensity could be derived from the pixel intensity of the light sources in the image. By employing a 3D mapping algorithm, or by measuring the distance to the ceiling with a laser diode, the height could also be determined. Finally, the evaluation of our approach was performed in a relatively small environment. Future work should verify whether the system performs the same in a larger environment.

\section{Acknowledgements}

Robin Amsters is an SB fellow of the Research Foundation Flanders (FWO) under grant agreement 1S57720N.

\section{References}

[1] Renub Research. Global led lighting market: Forecast by applications, regions and companies. Technical report, Renub Research.

[2] Steven De Lausnay, Lieven De Strycker, Jean Pierre Goemaere, Bart Nauwelaers, and Nobby Stevens. A survey on multiple access Visible Light Positioning. 2016 IEEE International Conference on Emerging Technologies and Innovative Business Practices for the Transformation of Societies, EmergiTech 2016, pages 38-42, 2016.

[3] Ye-sheng Kuo, Pat Pannuto, Ko-jen Hsiao, Prabal Dutta, and Ann Arbor. Luxapose : Indoor Positioning with Mobile Phones and Visible Light. Mobicom'14, pages 299-301, 2014.

[4] Trong-Hop Do and Myungsik Yoo. An in-Depth Survey of Visible Light Communication Based Positioning Systems. Sensors, 16(5):678, 2016.

[5] Sebastian Thrun, Wolfram Burgard, and Dieter Fox. Probabilistic robotics. MIT press, 2005.

[6] Joao Machado Santos, David Portugal, and Rui P. Rocha. An evaluation of 2D SLAM techniques available in Robot Operating System. 2013 IEEE International Symposium on Safety, Security, and Rescue Robotics, SSRR 2013, 2013.

[7] Wolfgang Hess, Damon Kohler, Holger Rapp, and Daniel Andor. Real-time loop closure in 2D LIDAR SLAM. Proceedings - IEEE International Conference on Robotics and Automation, 2016-June:1271-1278, 2016.

[8] Hui Liu, Houshang Darabi, Pat Banerjee, and Jing Liu. Survey of wireless indoor positioning techniques and systems. IEEE Transactions on Systems, Man, and Cybernetics, Part C (Applications and Reviews), 37(6):10671080, 2007.

[9] J. M. Lebreton, N. Murad, and R. Lorion. Real-time radio signal mapping using an autonomous robot. In 2015 Radio and Antenna Days of the Indian Ocean (RADIO), pages 1-2, Sep. 2015.

[10] P. Mirowski, R. Palaniappan, and T. K. Ho. Depth camera slam on a low-cost wifi mapping robot. In 2012 IEEE International Conference on Technologies for Practical Robot Applications (TePRA), pages 1-6, April 2012. 
[11] D. Hahnel, W. Burgard, D. Fox, K. Fishkin, and M. Philipose. Mapping and localization with rfid technology. In IEEE International Conference on Robotics and Automation, 2004. Proceedings. ICRA '04. 2004, volume 1, pages 1015-1020 Vol.1, April 2004.

[12] A. Milella, P. Vanadia, G. Cicirelli, and A. Distante. Rfid-based environment mapping for autonomous mobile robot applications. In 2007 IEEE/ASME international conference on advanced intelligent mechatronics, pages 1-6, Sep. 2007.

[13] Ivan Moreno and Ching-Cherng Sun. Modeling the radiation pattern of LEDs. Optics express, 16(3):1808-19, 2008.

[14] Fakhrul Alam, Baden Parr, and Susan Mander. Visible Light Positioning Based On Calibrated Propagation Model. IEEE Sensors Letters, PP(3):1-1, 2018.

[15] Yang Qiu, Hsiao-Hwa Chen, and Wei-Xiao Meng. Channel modeling for visible light communications—a survey. Wireless Communications and Mobile Computing, (February 2015):421-430, 2016.

[16] Radu Bogdan Dragomir, Radu Dragomir, Introduction Applications, European Union, and The Led. Irradiance model and simulation of a lighting led system. 76, 2014.

[17] Mark Halper. Robot helps light Japan's Toyota Stadium, 2019.

[18] OpenMV. OpenMV Cam M7 I OpenMV, 2019.

[19] Christos Danakis, Mostafa Afgani, Gordon Povey, Ian Underwood, and Harald Haas. Using a CMOS camera sensor for visible light communication. 2012 IEEE Globecom Workshops, GC Wkshps 2012, pages 1244-1248, 2012.

[20] Mohammed Khider, Susanna Kaiser, Patrick Robertson, and Michael Angermann. The effect of maps-enhanced novel movement models on pedestrian navigation performance. In Proceedings of The 12th annual European Navigation Conference (ENC 2008), Toulouse, France, volume 2228, 2008.

[21] Canny John. A computational approach to edge detection. IEEE Transactions on Pattern Analysis and Machine Intelligence, 8(6), 1986.

[22] Guiseppe Conti, Fabio Malabocchia, Ki-Joune Li, George Percivall, Kirk Burroughs, and Stuart Strickland. Benefits of Indoor Location, Use Case Survey of Lessons Learned and Expectations. Technical report, 2016. 